\title{
Themenheft „Hydrogeologie der Festgesteine“
}

\author{
Hartmut Holländer ${ }^{1}$ Ingo Bardenhagen ${ }^{2}$
}

Angenommen: 30. Dezember 2021 / Online publiziert: 20. Januar 2022

(c) The Author(s), under exclusive licence to Springer-Verlag GmbH Deutschland, ein Teil von Springer Nature 2022

Auf etwa 46\% der Fläche Deutschlands stehen mehr oder weniger ergiebige Festgesteine an, die lokal bis regional für die Wasserversorgung genutzt werden. In vielen dieser Regionen wurde im vergangenen Jahrhundert die lokale bis regionale Wasserversorgung aus Festgesteinsleitern mehr und mehr zurückgebaut und durch zentrale Wasserversorgungen aus Oberflächengewässern (Stauseen, Bodensee) etc. ersetzt. Wie vulnerabel zentrale Wasserversorgungen sind, zeigte nicht zuletzt der Giftanschlag auf die Bodenseewasserversorgung 2005, der viele Gemeinden, selbst in Regionen mit ungünstigen Festgesteinsleitern, zum Umdenken bewegte, stillgelegte Quellfassungen und Brunnen wieder in Betrieb zu nehmen - nicht zuletzt auch um Kosten zu sparen. Viele dieser Gemeinden fahren seither zweigleisig.

Die Herausforderung, Wasserressourcen im Festgestein zu erschließen, besteht darin, dass das Grundwasser im Festgestein im diskret - zum Teil erratisch - verteilten Trennflächengefüge fließt. Form und Ausprägung des Trennflächengefüges werden durch die Gesteinseigenschaften und die tektonische Beanspruchung der Gesteine geprägt. Wichtigste Grundlage einer hydrogeologischen Charakterisierung von Grundwasserleitern im Festgestein ist daher eine strukturgeologische Analyse bzw. Beschreibung, um Bilanzräume festzulegen, Einzugsgebiete zu lokalisieren oder Bohrpunkte möglichst in höffigen Bereichen zu platzieren. Bitzer et al. kommen zu dem Schluss, dass der Buntsandsteingrundwasserleiter im Raum Grünstadt, trotz seiner tektonisch bedingten Komplexität, mithilfe von Altersdatierungen in Verbindung mit Abschätzungen zur Fließrichtung in bilanzierbare hydrogeologische Teilräume

Hartmut Holländer

hartmut.hollaender@umanitoba.ca

Ingo Bardenhagen

ingo.bardenhagen@lbeg.niedersachsen.de

1 Department of Civil Engineering, University of Manitoba, Winnipeg, Kanada

2 Landesamt für Bergbau, Energie und Geologie Geozentrum Hannover, Hannover, Deutschland gegliedert werden kann. Die Bedeutung von natürlichen Quellen und bergbaulich angelegten Strukturelementen, wie z.B. Stollen für die Rekonstruktion der zu erwartenden Druckfläche nach einer langjährigen bergbaulichen Grundwasserabsenkung, zeigen Ottenjann et al.

Die Leistung und das gewinnbare Dargebot von Brunnen im Festgestein hängt von der Konnektivität und der Öffnungsweite des offenen Trennflächengefüges ab. Bei größeren Öffnungsweiten fungieren einzelne Trennflächen als bevorzugte Fließwege („Conduits“), deren Durchlässigkeit im Vergleich zur Durchlässigkeit der umgebenden Gesteinsbereiche, d.h. der Matrix, nahezu ,infinit“ ist. In solchen Fällen kann die Brunnenleistung erheblich über der eines Porengrundwasserleiters mit vergleichbarer Matrixdurchlässigkeit liegen. Ein Beispiel hierfür wird von Vassolo et al. in ihrem Beitrag beschrieben.

Das gewinnbare Dargebot ist im Wesentlichen von der Grundwasserneubildung und dem speicherfähigen Trennflächenvolumen abhängig. Beides ist in einem Festgesteinsleiter meist erheblich geringer als in typischen Porengrundwasserleitern, weil das Eindringen von Niederschlagswasser aufgrund der sporadischen Trennflächenverteilung reduziert ist und das Trennflächenvolumen in der Regel deutlich geringer ist als das Porenvolumen eines sandig-kiesigen Leiters. Wie dennoch die Grundwasserneubildung abgeschätzt werden kann, stellen Ertl et al. anhand einer erweiterten Methode basierend auf mGROWA für fünf Mittelgebirgslandschaften in Südniedersachsen vor.

Obwohl das Trennflächengefüge das dominante Strukturelement in Festgesteinsleitern ist, sind gerade für den Stofftransport und die chemischen Reaktionsräume die ungeklüftete Gesteinsmatrix bzw. der Kontakt zwischen Gesteinsoberfläche und Trennfläche von Bedeutung. Maßgeblich für den Stofftransport ist die Gesteinsdurchlässigkeit. Hale und Blum beschreiben die Bestimmung des Gesteinsdurchlässigkeitsbeiwertes mit einem Luftpermeameter, während Thielen und Rüde neue Erkenntnisse aus einem Modellversuch über die hydraulischen Eigenschaften und den Stofftransport in der Kontaktzone Kluft-Gesteins- 
matrix im karbonatisch gebunden Luxemburger Sandstein vorstellen.

Trotz zahlreicher Fortschritte, z. B. bei der oberflächennahen Strukturanalyse durch Scherwellenseismik, bei bildgebenden Verfahren in Bohrungen, bei hochauflösenden fotogrammetrischen Vermessungen von Trennflächengefügen in Steinbrüchen oder bei der Berechnung von Trennflächenverteilungsmustern, ergibt die Charakterisierung eines Festgesteinsleiters nach wie vor nur ein grobes Abbild der „Wirklichkeit“ verglichen mit den Modellvorstellungen in Porengrundwasserleitern. Hier wäre es wünschenswert, kostengünstigere Verfahren zu entwickeln, die eine flächendeckende strukturgeologische Erkundung oberflächennaher Grundwasserleiter ermöglichen.
Verbesserte Strukturanalysen werden jedoch Feldmessungen, wie Quellschüttungsmessungen, geohydraulische Feldversuche, Tracerversuche und Grundwasserstandsmessungen sowie regelmäßige Beprobungen für chemische und isotopenchemische Untersuchungen nicht überflüssig machen. Feldmessungen werden nach wie vor wichtige Eckdaten für die hydrogeologische Charakterisierung von Festgesteinen liefern.

Hinweis des Verlags Der Verlag bleibt in Hinblick auf geografische Zuordnungen und Gebietsbezeichnungen in veröffentlichten Karten und Institutsadressen neutral. 